www.jmscr.igmpublication.org Impact Factor 5.84

Index Copernicus Value: 83.27 ISSN (e)-2347-176x ISSN (p) 2455-0450 crossref DOI: _https://dx.doi.org/10.18535/jmscr/v5i8.106

Journal Of Medical Science And Clinical Research

\title{
Presenting A Case of Supra Renal Mass in Young Child - A Case Report
}

Authors

\author{
Dr Anil Kumar Jangir ${ }^{1}$, Dr Chaturvedy Kirti Rana ${ }^{2}$, Dr R.N. Gehlot ${ }^{3}$ \\ Department of Radiodiagnosis, Dr.S.N.M.C, Jodhpur (Raj)
}

\section{Aims and Objectives}

This case report aims to imaging spectrum in case of neuroblastoma.

\section{Case Report}

- A 4 yr male child presented with c/o body ache, swelling on scalp region since 3 month of age, pain abdomen and fever from last 1 month.

- GPE - cachexia and raised ICP signs

- Blood investigations are- $\mathrm{Hb}-2.7 \mathrm{gm} \%$, TLC - 9800, platelets - 1.2 lakh

\section{○ ESR 140}

- FNAC report shows - possibility of histiocytic disorder (nodal langerhans cell histiocytosis is suggested)

- Bone marrow aspiration: few scattered myeloid and erythroid series cells are seen.

- X ray skull is performed.

- X ray skull shows Osteolytic lesion, hair on end appearance and diffusely widened skull sutures.
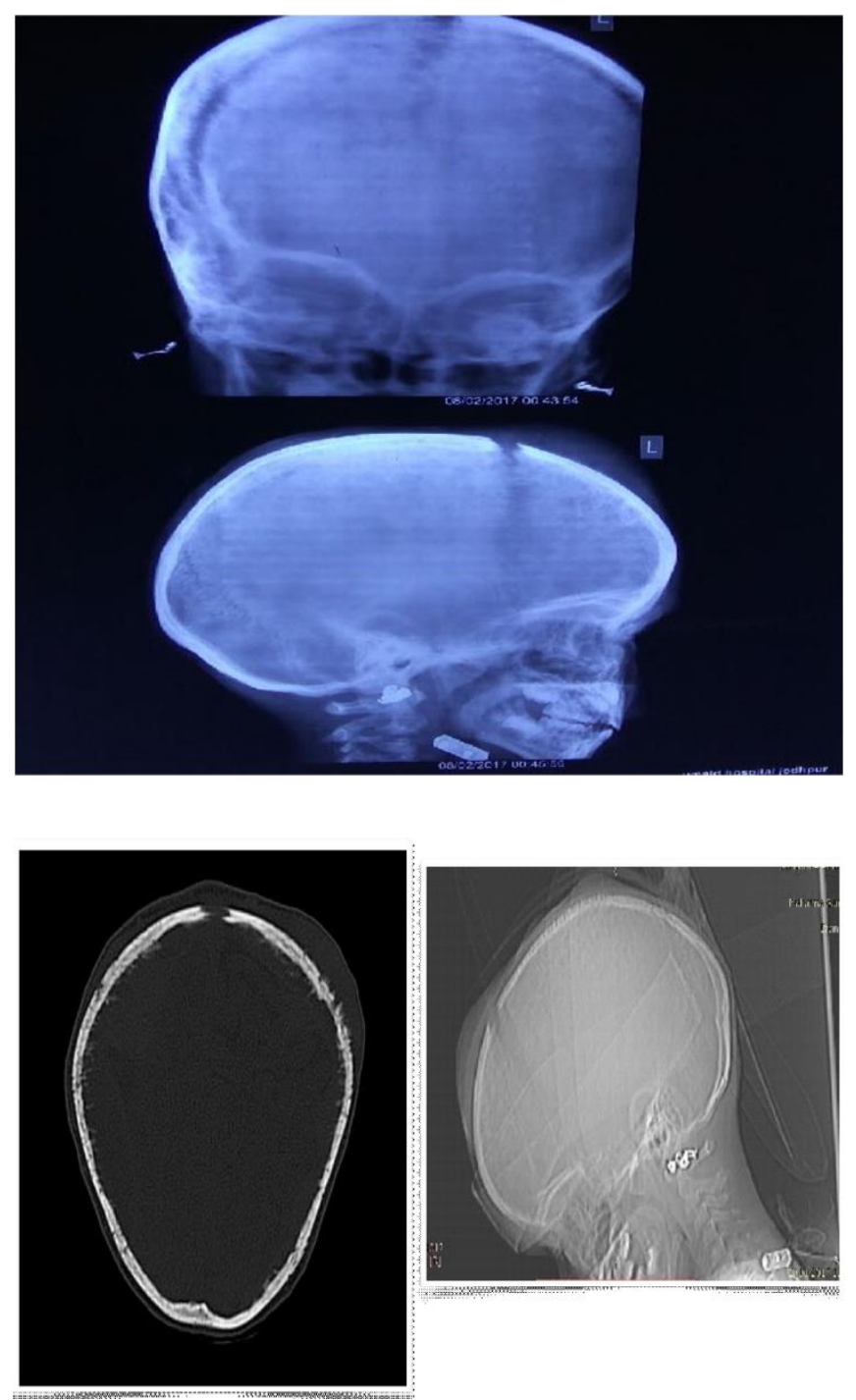

Surview Images 
-diffuse moth eaten osteolytic lesion - sun ray spiculations
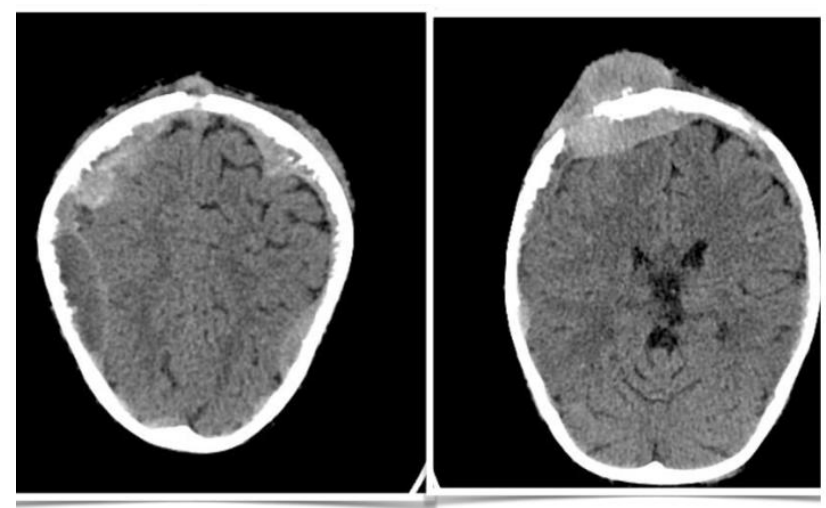

NCCT Brain images
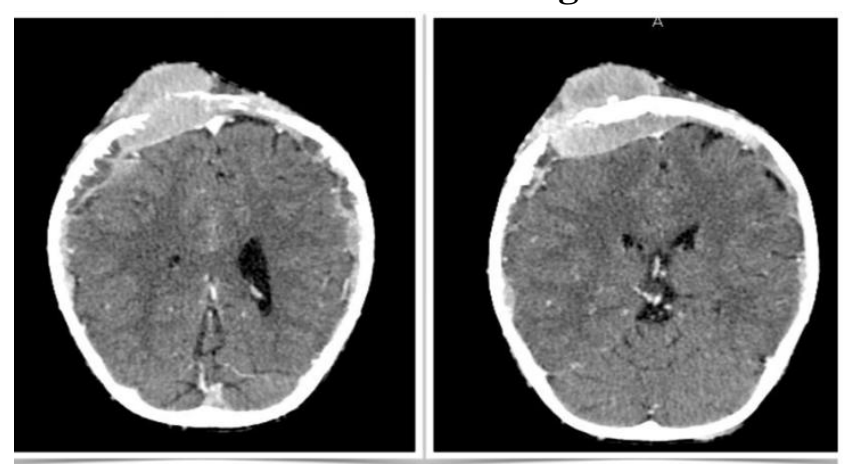

CECT brain images

On CECT BRAIN there is hyper dense mildly and heterogeneously enhancing nodular and extraaxial (subdural) as well as extra cranial mass lesion seen along $b / 1$ fronto-parieto-occipital convexity with sunray spiculations and diffuse moth eaten osteolytic lesion with diffusely widened skull sutures also seen.

On USG abdomen there is a well defined large mixed echoic mass lesion of size $6.5 \times 5 \mathrm{~cm}$ with internal calcification and hypo echoic area noted in supra renal region of left kidney.

-Hepatosplenomegaly *

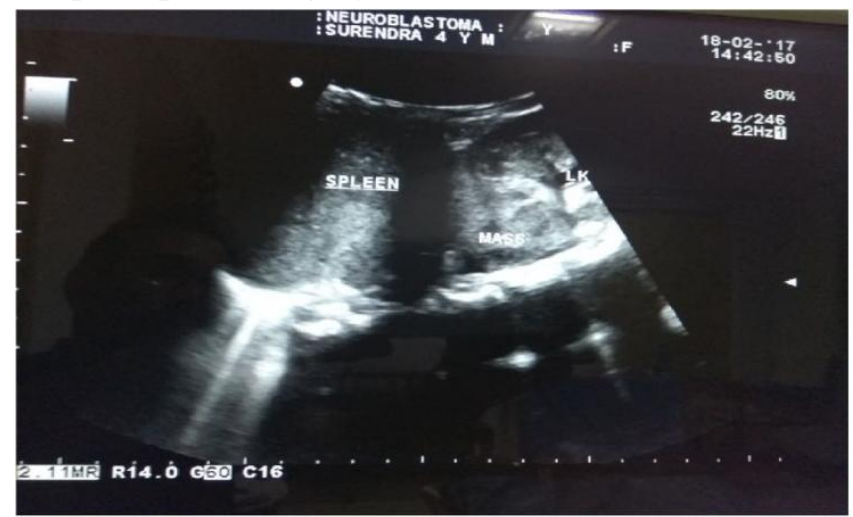

USG image of Patient
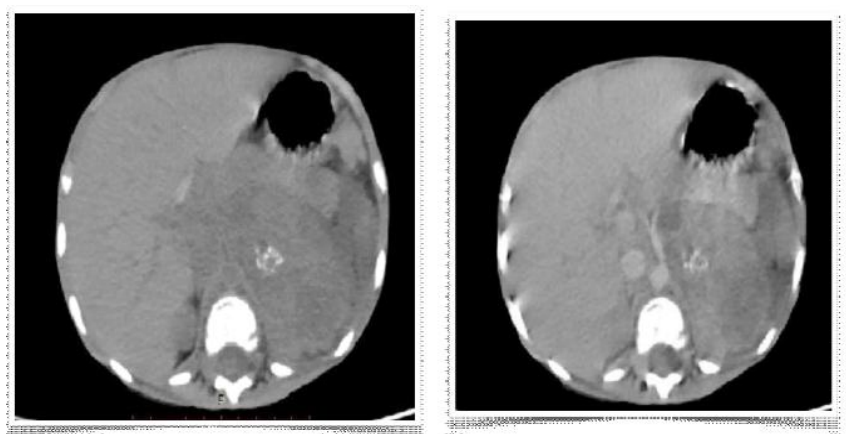

plain and contrast axial images $\mathrm{CT}$.

CECT abdomen shows a large well defined heterogeneously enhancing solid mass lesion measuring $\sim 7.8 \times 5.0 \times 6.5 \mathrm{~cm}$ with internal non enhancing areas and flakes of calcification seen in left supra renal region.

-This mass lesion causing displacement of pancreas and stomach to anteriorly and left kidney to inferolaterally. The mass causing encasement of splenic artery.

-Mass does not cross midline.

\section{Discussion}

Neuroblastoma is the third most common malignancy in children after leukemia and primary CNS brain tumours.

The tumours typically occur in infants and very young children with $90 \%$ of cases diagnosed before the age of 5 years.

Common sites - neuroblastoma can occur anywhere along sympathetic chain or in adrenal gland. other sites- posterior mediastinum, pelvis, neck.

The most common clinical presentation of this tumour is abdominal mass, however patient

may present with wide range of symptoms that results from excess hormone production or mass effect of primary tumor or metastasis, may have palpable mass, abdominal pain or hypertension from compression of renal artery.

$2 / 3 \mathrm{rd}$ patients have metastatic disease at time of diagnosis.

Metastatic involvement of the skull produces several possible radiographic findings:

thickened bone, the so-called "hair-on-end" periosteal reaction, lytic defects, and separation of 
sutures. The differential diagnosis of multiple lytic skull lesions in a child includes - Langerhans cell histiocytosis, leukemia, lymphoma, sarcoma, metastases

\section{USG}

Neuroblastoma appears as a solid, echogenic, usually heterogenous mass. may show calcification hemorrhage or cystic changes.

\section{CT}

On CT, the tumour typically is heterogeneous with calcifications seen in $80-90 \%$ of cases.

Areas of necrosis are of low attenuation.

The tumour tends to encase vessels and may lead to compression. (most characteristic imaging feature)

Adjacent organs are usually displaced, although in more aggressive tumours direct invasion of the psoas muscleor kidneycan be seen.In the latter, it can make distinguishing neuroblastoma from Wilms tumourdifficult.

Lymph node enlargementis often present.

$99 \mathrm{~m} \mathrm{Tc}$ MDP scintigraphy is useful for detection of bone metastasis

\section{MRI}

Modality of choice for evaluation of abdominal neuroblastoma.

MRI is superior to all other modalities in assessing the organ of origin, intracranial or intraspinal disease and bone marrow disease

- T1: heterogeneous and iso to hypointense

- $\mathrm{T} 2$

- heterogeneous and hyperintense

- cystic/necrotic areas very high intensity

- signal voids may be evident

- $\mathrm{C}+(\mathrm{Gd})$ : variable and heterogeneous enhancement

\section{Treatment}

Surgery

Chemotherapy

Bone marrow transplantation

International neuroblastoma staging system
Stage
description

1 Localized tumor confined to the area of origin; complete gross excision, with or without microscopic residual disease; identifiable ipsilateral and contralateral lymph nodes negative microscopically

2A

Unilateral tumor with incomplete gross excision; identifiable ipsilateral non adherent lymph nodes negative microscopically

2B Unilateral tumor with complete or incomplete gross excision; positive ipsilateral non adherent lymph nodes; identifiable contralateral lymph nodes negative microscopically

3 Tumor infiltrating across the midline (vertebral column) with or without regional lymph node involvement; or unilateral tumor with contralateral regional lymph node involvement; or midline tumor with bilateral regional lymph node involvement or extension by infiltration

4 Dissemination of tumor to distant lymph nodes, bone, bone marrow, liver, or other organs (except as defined in stage 4S)

4S Localized primary tumor as defined for stage 1 or 2 with dissemination limited to liver, skin and/or bone marrow ( $10 \%$ tumor) in infants younger than $1 \mathrm{y}$

\section{References}

1. David R, Lamki N, Fan S, Singleton EB, Eftekhari F, Shirkhoda A, et al. The many faces of neuroblastoma. Radiographics 1989;9:859-82

2. Maris JM, Hogarty MD, Bagatell R, Cohn SL. Neuroblastoma. Lancet 2007;369: 2106-20

3. De Bernardi B, Nicolas B, Boni L, Indolfi P, Carli M, Cordero DiMontezemolo L, et al. Disseminated neuroblastoma in children older than one year at diagnosis: comparable results with three consecutive high-dose protocols adopted by the Italian Co-Operative Group for Neuroblastoma. J Clin Oncol 2003;21:1592-601

4. Joshi VV. Peripheral neuroblastic tumors: pathologic classification based on recommendations of international neuroblastoma pathology committee 
(modification of Shimada classification). Pediatr Dev Pathol 2000; 3:184 -199.

5. Brodeur GM, Pritchard J, Berthold F, et al. Revi- sions of the international criteria for neuroblastoma diagnosis, staging, and response to treatment. J Clin Oncol 1993; 11:1466 - 1477.

6. Pearson ADJ, Pinkerton R. Neuroblastoma. In: , Pinkerton R, Plowman PN, Pieters R. eds. Paediatric oncology. London, England: Arnold, 2004; 386-414. 